\title{
A Simple Method of the $\mathrm{T}_{\mathrm{E}} \mathrm{X}$ Surface Drawing Suitable for Teaching Materials with the Aid of CAS
}

\author{
Masataka Kaneko, Hajime Izumi, \\ Kiyoshi Kitahara $^{1}$, Takayuki Abe, Kenji Fukazawa ${ }^{2}$, \\ Masayoshi Sekiguchi, Yuuki Tadokoro, \\ Satoshi Yamashita, and Setsuo Takato ${ }^{3}$ \\ ${ }^{1}$ Kisarazu National College of Technology, Japan \\ Kogakuin University, Japan \\ ${ }^{2}$ Kure National College of Technology, Japan \\ ${ }^{3}$ Toho University, Japan \\ nkaneko@inc.kisarazu.ac.jp
}

\begin{abstract}
The authors have been developing KETpic as a bundle of macro packages for Computer Algebra Systems (CASs) to draw fine TEX-pictures. Recently we have developed a new method of the surface drawing using KETpic. The equation of envelopes is used to draw ridgelines of surfaces. Also the technique of hidden line elimination is used. By these methods, we can draw 3D-graphics which are so simple that the global (i.e. sketchy) shapes of them are easily understood.
\end{abstract}

\section{Introduction}

We have been developing KETpic as a bundle of macro packages for CASs. It has been developed for inserting fine (accurate and expressive) figures into $\mathrm{LT}_{\mathrm{E} X}$ documents. We can readily produce a graphic output within such CASs and export the resulting figures to $\mathrm{LT}_{\mathrm{E} X} \mathrm{X}$ documents as source code rather than a graphical file itself. It is downloadable for free from [5].

Since KETpic has been equipped with various commands or accessories, its 2D-graphics have been actually used in our mathematics classroom. We can also draw space curves easily with KETpic. The 3D-graphics of KETpic are monochrome and are composed of lines.

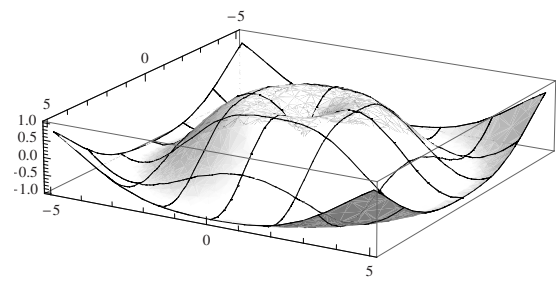

Fig. 1. The graphic of CAS

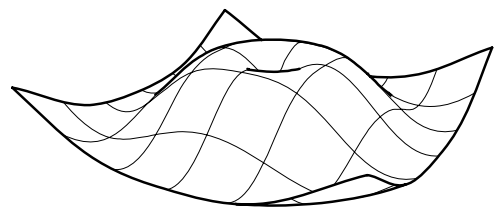

Fig. 2. The graphic of KETpic 
It takes little cost for us to make copies of the figures drawn with KETpic. Moreover, the quality of the figures is maintained when they are copied. Using these printed materials repeatedly, students can establish the mathematical concepts. Thus, they are suitable for teaching materials in the form of mass printed matter. On the other hand, the 3D-graphics of CASs are colourful and illuminated, so they are suitable for demonstration on displays. However, it takes much cost to maintain the quality of the graphic images of them when they are copied. Therefore, it is difficult for students to use them repeatedly.

Currently KETpic is equipped with the ability to eliminate the part of curves hidden by the other ones [1. We call such a technique "skeleton method". By using only one command "skeletondata" of KETpic, we can easily give clear perspective to 3D-graphics as shown in Fig. 3. These figures were actually used to explain the definition of double integral and the concept of repeated integral in our mathematics classroom.
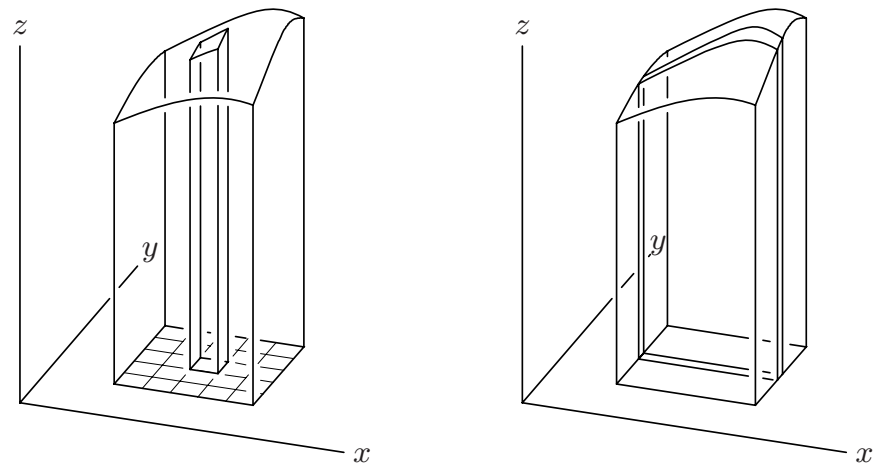

Fig. 3. Figures drawn with skeleton method

Though the ability of KETpic for drawing space curves is exploited as above, there were some deficits for surface drawing. In this paper, the authors introduce a new capability of KETpic for surface drawing. It meets the potential demands of the mathematics teachers who usually use CAS and $\mathrm{T}_{\mathrm{EX}}$, and who

1. want to show students the figures of surfaces with the accurate ridgelines just as we see them.

2. want to draw such figures using the minimal number of lines so as to make them easy to see.

In fact, a method to draw accurate ridgelines has been known. In that method, a point on the ridgelines is characterized by the following condition:

The normal vector of the surface at that point is orthogonal to the line connecting that point with the view point.

Since the equation describing this condition is simple, we can solve it by hand. However, this method is ad hoc in a sense, and it would be complicated in case of 
the surface given by parameters other than $x, y$. The method introduced in this paper is applicable to such general cases. Though the equation of our method is a little complicated and can not be solved by hand, it can be solved within almost the same time as in the above method by using CAS.

The authors also introduce the method to eliminate the parts of ridgelines hidden by the parts of the surface on this side of the viewer.

In the next Section 2, we introduce mathematical background of our method. In Section 3, we explain the procedure to draw surfaces using our method with an example.

\section{Mathematical Background}

\subsection{Setting of the Problem}

Suppose $z=f(x, y)$ is a smooth function with two variables defined on a domain $D$ in $\mathrm{R}^{2}$. When we draw a graph of $f$ on a plane, we actually draw its image under the projection $p$ onto the plane (the field of our view). The projection depends on the viewpoint and the focus point, and generally has a form of nonlinear transformation. The 3D-graphics of CAS are drawn also by using the automatic calculation of this projection $p$. Therefore, it should be impossible for us to draw a precise figure of the graph of $f$ without using CAS or other graphic softs.

In the above setting, the composition $p \circ f: \mathrm{R}^{2} \longrightarrow \mathrm{R}^{2}$ becomes a smooth map. For exactness, we give coordinate $(x, y)$ to the former $\mathrm{R}^{2}$ (containing domain $D$ ) and coordinate $(X, Y)$ to the latter $\mathrm{R}^{2}$ (containing image $(p \circ f)(D)$ ).

For example, we consider the graph of the function:

$$
z=2-x^{2}-y^{2} \quad(-1 \leq x \leq 1,-1 \leq y \leq 1) .
$$

It is drawn with CAS, and is inserted into $\mathrm{LT}_{\mathrm{E} X} \mathrm{X}$ document by using KETpic. Remark that Fig. 4 is drawn on the $X Y$-plane.

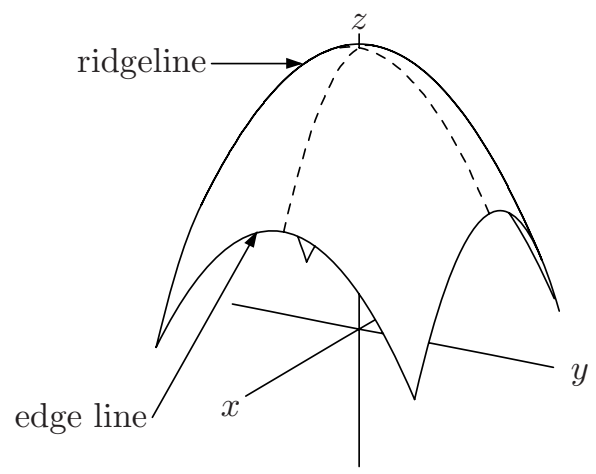

Fig. 4. Edge lines and ridgelines 
As shown in Fig. 4, the boundary of $(p \circ f)(D)$ in the $X Y$-plane is composed of two kinds of parts. One part is the set of "edge lines", the part of $(p \circ f)(\partial D)$. The other part is the set of "ridgelines".

Though this surface is obtained by the rotation of a parabola, the ridgeline is not a parabola. As is easily seen, it is nothing but the envelope of the image of some family of curves on the surface. We can draw edge lines easily by using CAS. On the other hand, it is not so easy to draw ridgelines.

\subsection{Main Result}

In this subsection, we show a method to draw ridgelines by using CAS.
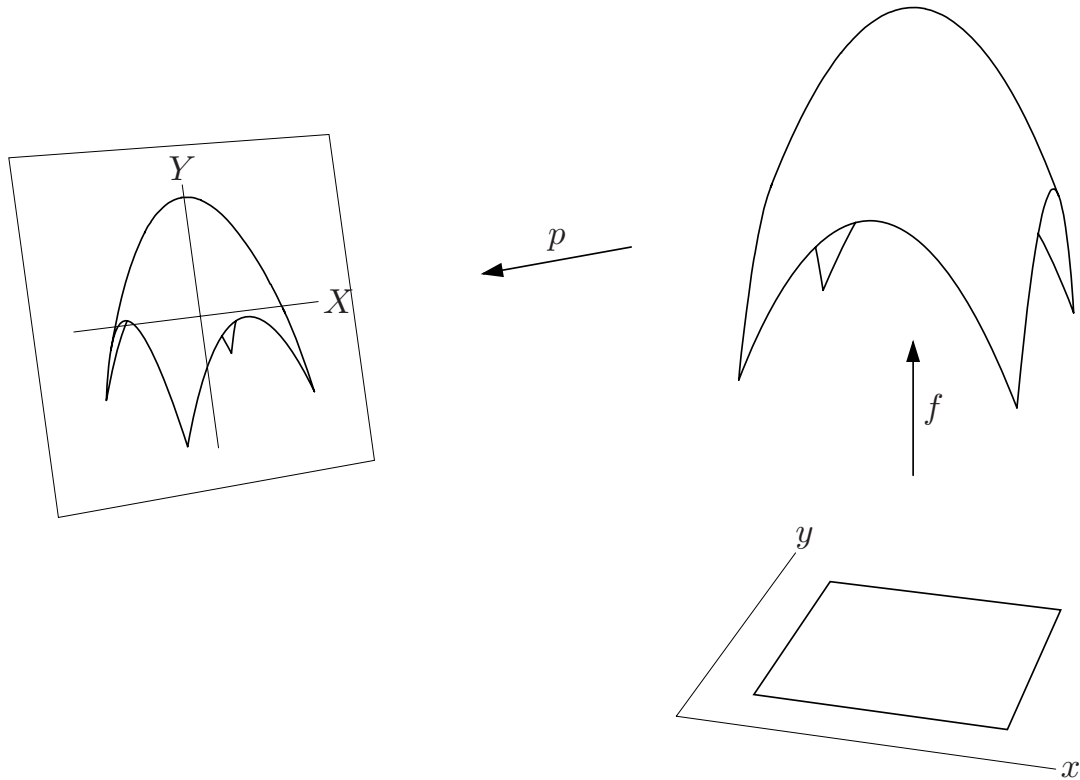

Fig. 5. Setting of the main result

We represent $p \circ f$ by components as follows:

$$
(p \circ f)(x, y)=\left(F_{1}(x, y), F_{2}(x, y)\right) .
$$

Then our main result is the next

\section{Proposition}

If the point $\left(F_{1}(x, y), F_{2}(x, y)\right)$ is located on the ridgelines of the graph of $f$, then the following equality holds at $(x, y)$ :

$$
\frac{\partial F_{1}}{\partial x} \frac{\partial F_{2}}{\partial y}-\frac{\partial F_{1}}{\partial y} \frac{\partial F_{2}}{\partial x}=0 .
$$




\section{Proof}

Firstly, remark that, to deduce the equation of the envelope, it is sufficient to consider any family of curves on the image of $p \circ f$ the union of which covers the image of $p \circ f$. So that, we can deduce the equation by using both of the following families of curves on the image:

$$
\left\{\begin{array} { l } 
{ X = F _ { 1 } ( x , \beta ) } \\
{ Y = F _ { 2 } ( x , \beta ) }
\end{array} \quad \left\{\begin{array}{l}
X=F_{1}(\alpha, y) \\
Y=F_{2}(\alpha, y)
\end{array}\right.\right.
$$

Here $\alpha$ and $\beta$ indicate the parameters of the families.

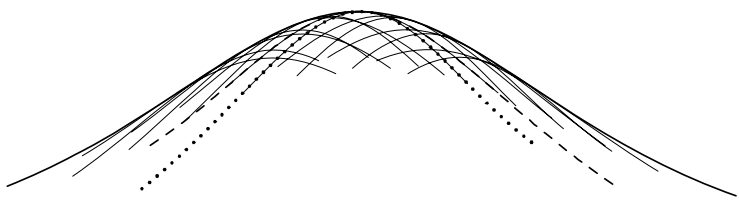

Fig. 6. Idea of proof

In Fig. 6 the dotted line is a member of $\alpha$-family, and the dash line is a member of $\beta$-family. Remark that these two lines are tangent to each other at one point of the envelope.

By the formula for the differentials of the functions with parametric representations, the slopes of the tangent lines to each of the above two families are equal to

$$
\frac{\partial F_{2}}{\partial x} / \frac{\partial F_{1}}{\partial x}, \quad \frac{\partial F_{2}}{\partial y} / \frac{\partial F_{1}}{\partial y}
$$

respectively. Clearly, at each point of the envelope, a curve belonging to $\alpha$-family is tangent to a curve belonging to $\beta$-family. Hence, at that point, the equality

$$
\frac{\partial F_{2}}{\partial x} / \frac{\partial F_{1}}{\partial x}=\frac{\partial F_{2}}{\partial y} / \frac{\partial F_{1}}{\partial y}
$$

holds. This implies the claim of our proposition.

\subsection{The Meaning of the Main Result}

As is easily seen, the quantity

$$
\frac{\partial F_{1}}{\partial x} \frac{\partial F_{2}}{\partial y}-\frac{\partial F_{1}}{\partial y} \frac{\partial F_{2}}{\partial x}
$$

is nothing but the Jacobian of the map $p \circ f: \mathrm{R}^{2} \longrightarrow \mathrm{R}^{2}$. Therefore, our proposition means that the envelope is given as the singular value set of the $\operatorname{map} p \circ f$.

In singular point theory, it is well known that the singular point set is given as the set of points where the Jacobian of the map vanishes. In our situation, 
the envelope corresponds to the singular value set of $p$ (defined on the image of $f$ ). Since $f$ is a diffeomorphism onto its image, the critical value set of $p \circ f$ is the same as that of $p$ (by the chain rule of Jacobian).

From the viewpoint of singular point theory, it seems to be meaningless to consider $p \circ f$ instead of $p$. Actually there is almost no opportunity of using $p \circ f$ because it is very difficult to give the explicit representation of it by hand. However, $p \circ f$ becomes very important when we draw figures by using CAS. This is because CAS can calculate $p \circ f$ and its differential. Furthermore, CAS can automatically solve the equation in the proposition.

Based on our main result, we can draw simple figures of surfaces with the minimal number of lines. The simpleness of the figures has not only aesthetic value but also mathematical meaning. In fact, it is an important idea of Morse theory 2 that the topological information of a manifold is contained in the structure of some critical submanifolds for a Morse function defined on the manifold.

\section{The Procedure to Draw Surfaces by Using KETpic}

In this section, we explain how to draw surfaces by using the method given in the previous section. Here we draw the graph of the following function as an example:

$$
f(x, y)=3\left(1-\left(2 x^{2}+y^{2}\right) e^{-\left(x^{2}+y^{2}\right)}\right) .
$$

\subsection{Calculate the Critical Point Set}

Firstly, we calculate the critical point set of $p \circ f$ which is a set of connected curves in the $x y$-plane. By fixing the focus point and the view point, the command such as "implicitplot" of CAS enables us to find the points which satisfy the equation in the previous section. Connecting the neighbouring points by segments, we can obtain the picture like Fig. 7. Remark that the number of connected components depends on the choice of the focus point and the view point.

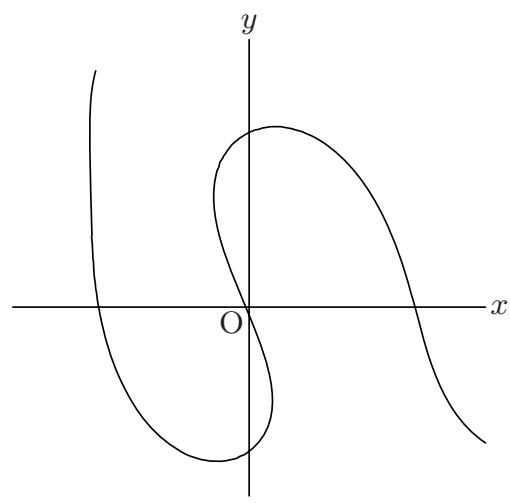

Fig. 7. Critical point set 


\subsection{Calculate the Critical Value Set}

Secondly, we draw the graph of $f$ restricted to the above critical point set. It is drawn in the $X Y$-plane as in Fig. 8. The ridgelines which we want to draw are composed of some parts of this critical value set.

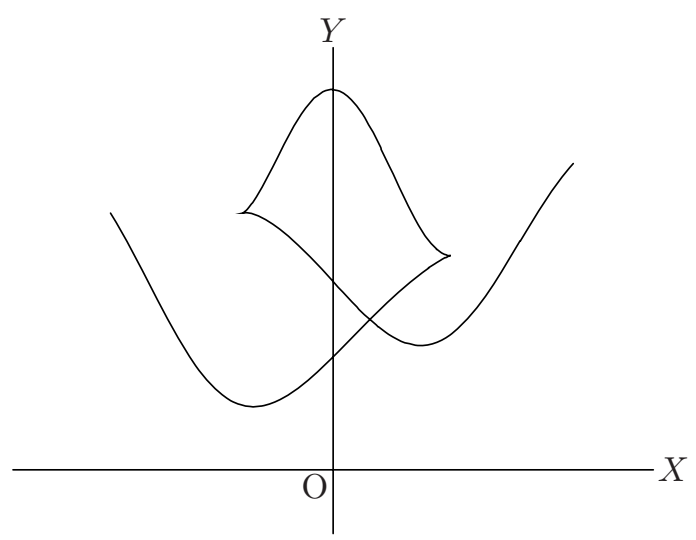

Fig. 8. Critical value set

There are some cusps in the critical value set. In Fig. 9, these points are specified clearly by using bold points. Topologically, the graph of $f$ is composed of three disks centered at extremal points of $f$. The cusps correspond to the points where the line segments bounding three disks are attached together.

We judge, by rather unrefined method, whether a point in the critical value set is a cusp or not. The judgement is accomplished by calculating the angle between the two vectors from that point to the neighbouring points.

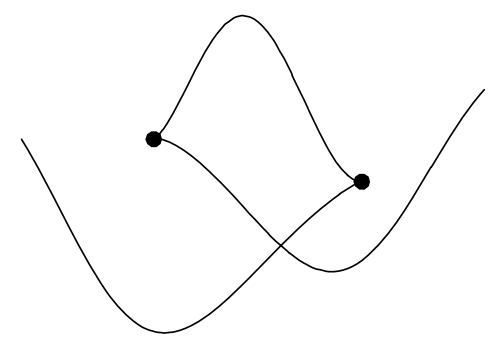

Fig. 9. Cusps

Though there are some parts in the critical value set which are invisible from the view point as shown in Fig. 11, they become visible when other parts on this side are eliminated. 


\subsection{Add Edges of the Graph and Eliminate Hidden Parts}

Thirdly, we add the edge lines of the graph to the critical value set. Then we obtain Fig. 10.

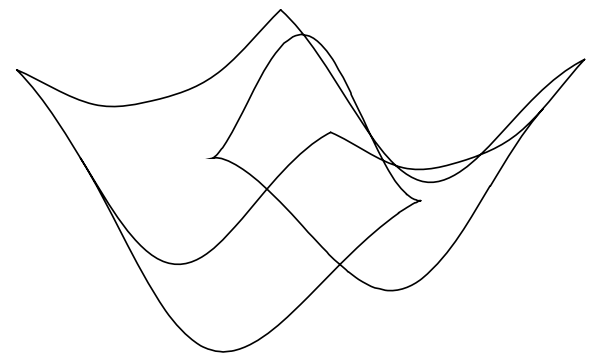

Fig. 10. Add edge lines

In these curves, there are some parts which are drawn in this picture but actually can not be seen from the viewpoint. So we must eliminate such hidden parts to draw the picture we want (as in Fig. 11).

As seen in Fig. 10, ridgelines and edge lines are separated to some parts by their own intersections or cusps. Remark that whether a point in these curves is visible or not is determined by the separated part to which the point belongs.

We judge whether a part is visible or not by following the next procedure:

1. Pick up an arbitrary point $\left(x_{0}, y_{0}, f\left(x_{0}, y_{0}\right)\right)$ from that part.

2. Take some points on the line connecting $\left(x_{0}, y_{0}, f\left(x_{0}, y_{0}\right)\right)$ with the view point by the method of bisection, and find the signature of $\{z-f(x, y)\}$ for each of these points.

3. If the signatures in the second step are constant, then the part containing $\left(x_{0}, y_{0}, f\left(x_{0}, y_{0}\right)\right)$ is visible. Otherwise, the part is invisible.

Thus, through this process of "hidden line elimination", we obtain the next Fig. 11 which we aimed at.

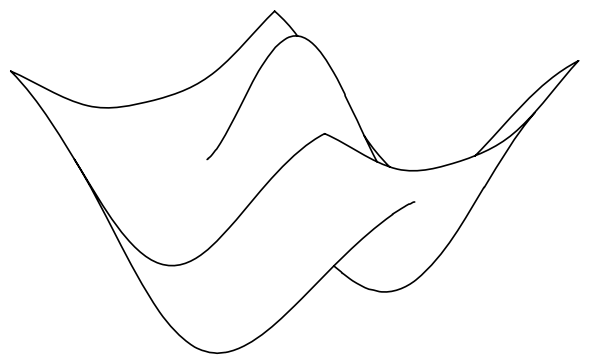

Fig. 11. Eliminate hidden lines

Even though this figure is precise with the aid of CAS, there remains only minimal information about the shape of the graph of $f$. This is because the 
minimal number of lines are used. Therefore, this figure enables us to grasp the global shape easily.

\subsection{Draw Wire Frames}

When we attach importance to the preciseness of the figure, we can also use wire frames widely used in 3D-graphics of CAS. Our graphics are more beautiful than those of CAS because the number of wires is small and because wires are smooth curves. In the next Fig. 12, five wires are drawn in both $x$ and $y$ directions. Remark that the technique of hidden line elimination can be also applied to wires.

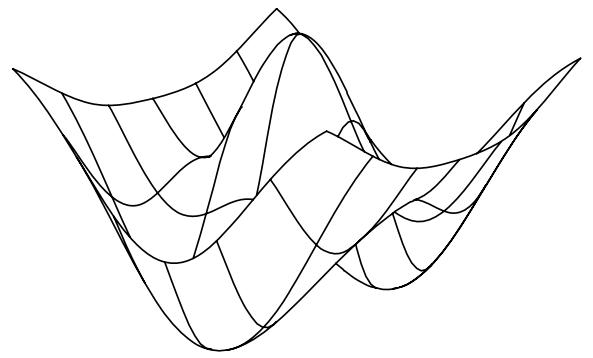

Fig. 12. Add wire frames

Compared with Fig. 11, Fig. 12 contains additional information about the local shape of the graph. In fact, the shape of the wire frames indicates the slope and curvature of the graph. In case of 3D-graphics of CAS, colours and half tone shadings indicate the local shape. Since it takes much cost for us to use colours and half tone shadings in teaching materials in the form of mass printings, the surface drawing of KETpic is more suitable for such practical use.

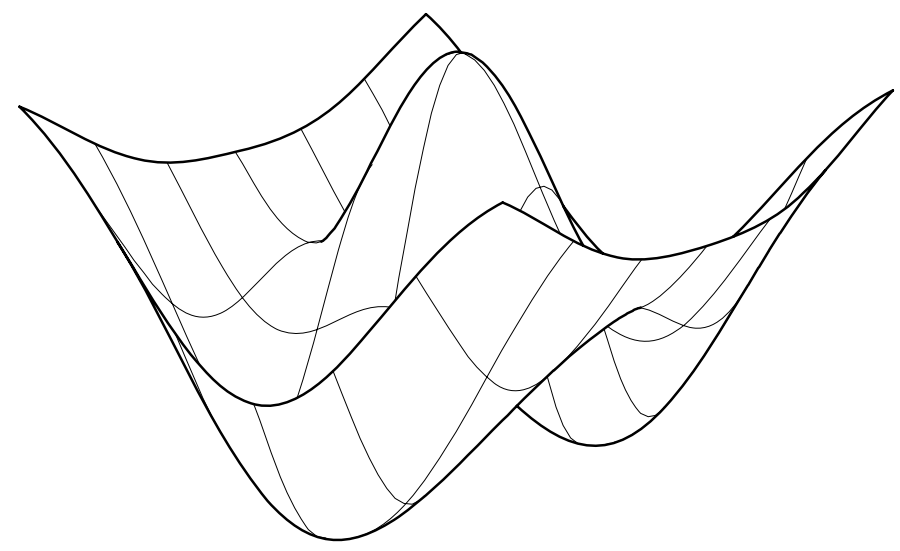

Fig. 13. Finished figure 
Oppositely, the existence of the wire frames makes it a little harder for us to grasp the global shape. This is because we must pick up the curves which are also contained in Fig. 11 from those in Fig. 12 to grasp the sketchy shape.

To make up this deficit, in Fig. 13, we draw clear distinction between the curves contained in Fig. 11 and wire frames by utilizing the ability of KETpic to draw thick and thin lines. This distinction makes this figure more easy to be understood. Thus, KETpic can offer 3D-graphics which are not only accurate (i.e. differential geometric) but also easy to understand (i.e. topological).

\section{Conclusion and Future Works}

The ability of surface drawing with minimal number of essential curves has great meaning. This is because we can easily understand the topological structure. As illustrated in the figures of this paper, we can draw accurate and simple 3Dgraphics in $\mathrm{T}_{\mathrm{E}} \mathrm{X}$ documents with KETpic. Moreover, KETpic enables us to hand the printed materials with those figures to students.

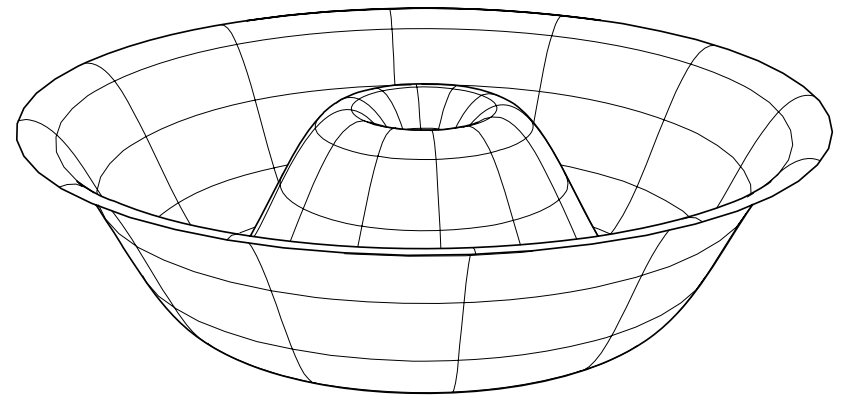

Fig. 14. The case of polar coordinate

The method which we have introduced in this paper can be applied to the case where the surface is given by parameters other than $x, y$ as follows:

$$
x=x(u, v), \quad y=y(u, v), \quad z=z(u, v) .
$$

Here we assume that the differential of the map $(u, v) \rightarrow(x, y, z)$ is injective.

The authors think that our method will be applicable also to the case of several surfaces. In the near future, we should develop a new KETpic functionality as above.

\section{References}

1. Kaneko, M., Abe, T., Sekiguchi, M., Tadokoro, Y., Fukazawa, K., Yamashita, S., Takato, S.: CAS-aided Visualization in LATEX documents for Mathematical Education. Teaching Mathematics and Computer Science (to appear)

2. Milnor, J.: Morse theory. Princeton Univ. Press, New Jersey (1963) 
3. Sekiguchi, M., Yamashita, S., Takato, S.: Development of a Maple Macro Package Suitable for Drawing Fine TEX-Pictures. In: Iglesias, A., Takayama, N. (eds.) ICMS 2006. LNCS, vol. 4151, pp. 24-34. Springer, Heidelberg (2006)

4. Sekiguchi, M., Kaneko, M., Tadokoro, Y., Yamashita, S., Takato, S.: A New Application of CAS to IATEX-Plottings. In: Shi, Y., van Albada, G.D., Dongarra, J., Sloot, P.M.A. (eds.) ICCS 2007. LNCS, vol. 4488, pp. 178-185. Springer, Heidelberg (2007)

5. Sekiguchi, M., http://www.kisarazu.ac.jp/ masa/math/ 